\title{
INGESTÃO DE ÁGUA NO SISTEMA NERVOSO AUTÔNOMO: UMA REVISÃO SISTEMÁTICA
} E META-ANÁLISE

\author{
WATER INTAKE EFFECT ON AUTONOMIC NERVOUS SYSTEM: A SYSTEMATIC \\ REVIEW AND META-ANALYSIS
}

INGESTIÓN DE AGUA EN EL SISTEMA NERVIOSO AUTÓNOMO: UNA REVISIÓN SISTEMÁTICA Y METAANÁLISIS

\author{
Thiago Casali Rocha \\ (Fisioterapeuta) \\ Plínio dos Santos Ramos ${ }^{2}$ \\ (Fisioterapeuta) \\ Djalma Rabelo Ricardo² \\ (Educador Físico) \\ 1. Universidade do Estado do Rio de \\ Janeiro (UERJ), Rio de Janeiro, \\ RJ, Brasil. \\ 2. Faculdade de Ciências Médicas e \\ da Saúde de Juiz de Fora, Hospital \\ e Maternidade Therezinha de Jesus, \\ Juiz de Fora, MG, Brasil.
}

\section{Correspondência:}

Programa de Pós-graduação em Ciências do Exercício e do Esporte da Universidade do Estado do Rio de Janeiro (UERJ), Rio de Janeiro, RJ, Brasil.

Rua: São Francisco Xavier, 524, Maracanã, Rio de Janeiro, RJ, 20550-900.rothi@oi.com.br

\section{RESUMO}

Verificar, por meio de uma revisão sistemática, o efeito da ingestão de água (IA) no sistema nervoso autônomo (SNA) e variáveis hemodinâmicas em indivíduos adultos. Foram analisados estudos publicados entre 2000 e 2015, tendo como referência a base de dados Medline via Pubmed, sendo utilizado na construção da frase de pesquisa o MeSH. Foram estabelecidos os seguintes critérios de inclusão: ensaios clínicos controlados e randomizados (ECCR) realizados em humanos, na língua inglesa. Como critério de exclusão: intervenções pouco claras, mal descritas ou inadequadas e na forma de resumos. Utilizou-se as seguintes variáveis para a seleção dos estudos: frequência cardíaca (FC), pressão arterial (PA), componente de alta frequência (AF) e resistência vascular periférica (RVP). Foi usada a sistematização PRISMA para a elaboração desta revisão e a realização de uma meta-análise com o objetivo de evidenciar matematicamente os resultados da frequência cardíaca após a ingestão de água em sete estudos que avaliaram esta variável. Fizeram parte desta revisão 10 ECCR envolvendo 246 indivíduos com idade entre 19 a 64 anos, sendo que 34,55\% do sexo masculino. A maioria dos ECCR analisados apresentou alterações após a IA. As alterações comumente observadas foram: diminuição da FC (estatisticamente significativa $p<0,001$ ), aumento da AF e RVP. Contudo, em relação à $\mathrm{PA}$, os resultados demonstraram-se conflitantes, com estudos que evidenciaram aumento e outros que não observaram diferença significativa. Esta revisão evidencia os efeitos da IA no SNA, em especial na FC, AF e RVP, não obstante em relação às alterações hemodinâmicas expressas pela PA permanece ainda um óbice em relação à comunidade científica.

Descritores: ingestão de líquidos, sistema nervoso autônomo, pressão arterial, frequência cardíaca, resistência vascular.

\section{ABSTRACT}

To verify, through a systematic review, the effect of water intake (WI) on the autonomic nervous system (ANS) and hemodynamic variables in adults. Studies published between 2000 and 2015 were analyzed, with reference to Medline database via Pubmed, and also MeSH was used in the construction of the search phrase. The following inclusion criteria were established: randomized and controlled clinical trials (RCTs) performed in humans, in English. Exclusion criteria: unclear, poorly described or inadequate interventions and in the form of abstracts. The following variables were used for selecting studies: heart rate (HR), blood pressure (BP), high frequency component (HF) and peripheral vascular resistance (PVR). The PRISMA systematization was used for the elaboration of this review and a meta-analysis was conducted in order to mathematically evidence the results of heart rate after water intake in seven studies that evaluated this variable. Ten RCTs were part of this review, involving 246 subjects aged 19-64 years, of whom 34.55\% were male. Most RCTs analyzed showed changes after WI. Changes commonly observed were the following: decreased HR (statistically significant $p<0.001$ ), increased $H F$ and PVR. However, in relation to BP results proved to be conflicting with studies that showed increase and others that showed no significant difference. This review shows the effect of WI on ANS, particularly in HR, HF and PVR, despite that, in relation to hemodynamic changes expressed by $B P$, an obstacle still remains regarding the scientific community.

Keywords: drinking, autonomic nervous system, arterial pressure, heart rate, vascular resistance.

\section{RESUMEN}

Verificar, a través de una revisión sistemática, el efecto de la ingestión de agua (IA) en el sistema nervioso autónomo (SNA) y las variables hemodinámicas en adultos. Fueron analizados estudios publicados entre 2000 y 2015, con referencia a la base de datos Medline vía PubMed, siendo utilizado en la construcción de la frase de búsqueda el MeSH. Se establecieron los siguientes criterios de inclusión: ensayos controlados y aleatorios (ECA), realizados en los seres humanos, en inglés. Criterios de exclusión: intervenciones poco claras, mal descritas o inadecuadas y en forma de resumen. Utilizamos las siguientes variables para la selección de los estudios: frecuencia cardíaca (FC), presión arterial (PA), componente de alta frecuencia (AF) y la resistencia vascular periférica (RVP). Se utilizó la sistematización PRISMA para la preparación de esta revisión y la realización de un metaanálisis con el fin de demostrar matemáticamente los 
resultados de la frecuencia cardíaca después de la ingestión de agua en siete estudios evaluando esta variable. Hicieron parte de esta revisión 10 ECA con 246 individuos de edades 19-64 años, siendo 34,55\% hombres. La mayoría de los ECA analizados mostraron cambios después de la IA. Los cambios observados comúnmente fueron: disminución de la FC (p estadísticamente significativa $<0,001$ ), el aumento de la AFy RVP. Sin embargo, en relación con la PA, los resultados demostraron ser contradictorios, con estudios que indicaron aumento y otros que no encontraron diferencias significativas. Esta revisión muestra los efectos de la IA en el SNA, en particular en la FC, AF y RVP, aunque en relación con los cambios hemodinámicos expresados por la PA sigue siendo un obstáculo en relación con la comunidad científica.

Descriptores: ingestión de líquidos, sistema nervioso autónomo, presión arterial, frecuencia cardíaca, resistencia vascular.

\section{INTRODUÇÃO}

O sistema nervoso autônomo (SNA) foi melhor descrito no início do século XIV', e é definido por estruturas com a função de inibir e excitar diferentes sistemas do corpo por dois ramos que trabalham em grande sintonia, chamados de sistema nervoso simpático e parassimpático². Desta forma, é uma temática amplamente estudada, por observar que alterações nesses sistemas apresentam fortes associações à morte súbita3 ${ }^{3-6}$.

É sabido que algumas variáveis podem ser capazes de influenciar a atividade dos ramos do SNA, tais como: medicações ${ }^{7}$, exercício físi$\mathrm{CO}^{8}$, respiração ${ }^{9}$, alimentação ${ }^{10}$ e outros. Sendo assim, o simples ato de ingerir água também tem assumido papel importante nas alterações hemodinâmicas e autonômicas do sistema cardiovascular ${ }^{11}$. Contudo, são vistas na literatura respostas antagônicas durante a ingestão de água (IA) para grupos de indivíduos com faixa etária e condições clínicas diferentes, como: em jovens saudáveis, idosos, hipertensos e também naqueles com disfunção autonômica ${ }^{12,13}$.

Já é demonstrado que o consumo de água tem se tornado um fator profilático e terapêutico para pacientes que sofrem de hipotensão ortostática ${ }^{14-16}$, e tendo em vista que o tratamento medicamentoso é dispendioso e o efeito bastante questionado ${ }^{17}$, percebeu-se a necessidade de encontrar os reais estímulos fisiológicos causados pela IA que induzem as respostas autonômicas, na qual duas vertentes vêm sendo alvo de maiores estudos para esclarecer tal fenômeno como a distensão gástrica ${ }^{18,19}$ e a osmolalidade 20,21 .

Nesse sentido, os efeitos comumente observados após a IA são: aumento da modulação vagal cardíaca ${ }^{22}$ e da pressão arterial ${ }^{14,23}$, bradicardia ${ }^{24}$ e aumento da resistência vascular periférica ${ }^{25}$. Diante disso, entende-se que o simples ato de ingerir água pode influenciar diretamente nos resultados dos estudos que pretendem avaliar as variáveis hemodinâmicas e autonômicas cardiovasculares. Todavia, permanece ainda um óbice na utilização da IA, tendo em vista que são verificados resultados conflitantes nos estudos que avaliam tal fenômeno ${ }^{12,13}$.

Entre as revisões sistemáticas existentes sobre a temática são comumente observados os estudos que exploram as recomendações de hidratação ${ }^{26,27}$ e os que elucidam as atuações das membranas plasmáticas de transporte, conhecidas como aquaporinas ${ }^{28,29}$. Porém, é observada escassez dentre os estudos de revisão com o intuito de esclarecer as possíveis modificações hemodinâmicas e autonômicas após a IA.

O objetivo do presente estudo, foi verificar por meio de uma revisão sistemática os efeitos da IA sobre as alterações hemodinâmicas e autonômicas cardiovasculares.

\section{MÉTODOS}

Foram analisados os mais relevantes estudos publicados originalmente, na língua inglesa, nos últimos 15 anos, tendo como referência a base de dados Medline. Objetivando os estudos com maior relevância clínica, foram escolhidos apenas os ensaios clínicos controlados e randomizados (ECCR).
O presente estudo utilizou para a formulação da frase de pesquisa as seguintes palavras-chave: Autonomic Nervous Systems; Parasympathetic; Heart Rate; WaterIntake, Blood Pressure. Para encontrar as variações das palavras-chave anteriormente apresentadas foi consultado o MeSH. Os critérios de inclusão e exclusão aplicados estão expostos no Quadro 1.

Quadro 1. Critérios de inclusão e exclusão.

\begin{tabular}{|c|}
\hline Critérios de inclusão \\
\hline Delineamento: Ensaios clínicos controlados e randomizados \\
\hline Intervenção: Ingestão de água \\
\hline Somente em humanos \\
\hline Indivíduos adultos com idade acima de 19 anos. \\
\hline Idioma: Língua inglesa \\
\hline Critérios de exclusão \\
\hline Intervenção: pouco clara, mal descrita ou inadequada \\
\hline Forma de publicação: somente resumos \\
\hline Principais variáveis analisadas \\
\hline Frequência cardíaca \\
\hline Pressão arterial \\
\hline Resistência vascular periférica \\
\hline
\end{tabular}

\section{Análise estatística}

Para os dados encontrados da FC foi realizada a meta-análise com o programa Medcalc 15.8, utilizando a análise estatística de efeito aleatório e efeito fixo, considerando a heterogeneidade dos estudos. O intervalo de confiança de 95\% (IC 95\%) foi calculado para cada estudo individualmente e em seguida, para a combinação dos estudos selecionados. Foi identificado à média e o desvio-padrão de cada estudo e adotado somente os valores de $p<0,05$ como significantes.

\section{RESULTADOS}

Foram identificados 8213 estudos envolvendo o sistema nervoso autônomo e IA. Contudo, a partir da aplicação dos critérios previamente definidos, apenas 136 fizeram parte do escopo desta revisão. Destes, apenas 10 foram elegíveis para fazerem parte do escopo desta revisão. A Figura 1 apresenta o fluxograma utilizado para a seleção dos artigos que foram analisados.

Os estudos analisados envolveram 246 voluntários com idade entre 19 a 64 anos, sendo 35\% do gênero masculino. A maioria dos ECCR apresentou resultados significativos após a IA entre as variáveis clínicas apresentadas. Um estudo que merece destaque objetivou avaliar as principais variáveis analisadas na presente revisão e encontrou diferenças significativas logo após a ingestão de $500 \mathrm{~mL}$ de água versus $50 \mathrm{~mL}^{14}$.

Em geral, as intervenções realizadas foram de curta observação, variando de 15 minutos a quatro semanas. Envolveram protocolos 


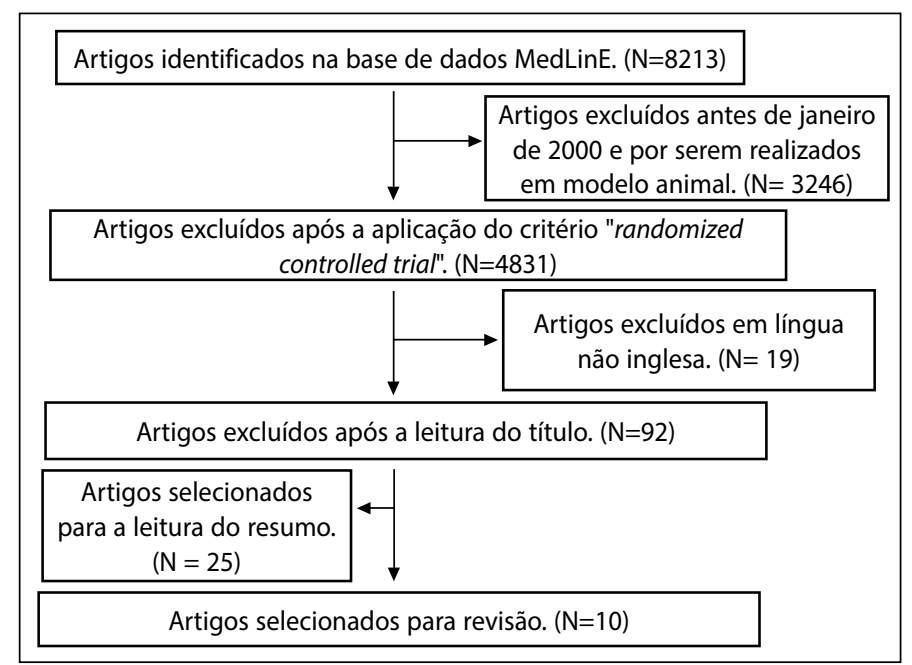

Figura 1. Fluxograma do processo de seleção dos estudos. altamente diversificados, tendo alguns alterando a quantidade de água ingerida, a temperatura e os testes para avaliações dos resultados autonômicos e hemodinâmicos.

Cabe ressaltar que grande parte dos estudos do escopo desta revisão, após a IA, apresentou tendência à redução da frequência cardíaca, aumento da resistência vascular periférica e resultados conflitantes sobre a pressão arterial. Já nos estudos que se propuseram avaliar a atividade vagal cardíaca, foi observado aumento significativo desta variável (Tabela 1).

\section{Meta-análise}

Apenas sete dos 10 estudos incluídos nesta revisão forneceram dados suficientes para analisar a FC após a ingestão de água 14,16,22,25,30-32. A meta-análise foi realizada com base nos sete artigos referidos, totalizando uma amostra com 166 voluntários. O valor da diferença da média padronizada foi de -0,815 e -1,110 para os efeitos fixos e aleatórios respectivamente.

Tabela 1. Sumário dos estudos e seus principais resultados para pressão arterial, frequência cardíaca, componente de alta frequência e resistência vascular periférica.

\begin{tabular}{|c|c|c|c|c|c|c|}
\hline \multirow{2}{*}{ Estudos } & \multirow{2}{*}{ Amostra } & \multirow{2}{*}{ Intervenção } & \multicolumn{4}{|c|}{ Variáveis analisadas } \\
\hline & & & PA & FC & AF & RVP \\
\hline \multirow{5}{*}{ Lu CC et al., $2012^{25}$} & 15 voluntários & Utilização de um cateter venoso para mensuração da FC, PA, RVP & & & & \\
\hline & Todos do sexo masculino & & $=P A$ & $=F C$ & NA & $R V P \uparrow$ \\
\hline & (Idade 19- 27 anos). & 60 minutos de observação & $p>0,01$ & $p>0,01$ & & $p<0,001$ \\
\hline & $\mathrm{GC}=50 \mathrm{ml}$ & & & & & \\
\hline & $\mathrm{Gl}=500 \mathrm{ml}$ & & & & & \\
\hline \multirow{4}{*}{ Chiang CT et al., $2010^{32}$} & 53 voluntários & $\begin{array}{c}\mathrm{GC}=250 \mathrm{ml} \text { temperatura } \\
\text { ambiente. }\end{array}$ & & & & \\
\hline & $\mathrm{GC}=25$ voluntários Idade: $(28.6 \pm 10.4)$ & $\mathrm{Gl}=250 \mathrm{ml}$ água gelada & NA & $\mathrm{FC} \downarrow$ & $\mathrm{AF} \uparrow$ & NA \\
\hline & & Os indivíduos foram avaliados através da VFC. & & $p<0.001$ & $p<0.001$ & \\
\hline & $\mathrm{Gl}=28$ voluntários Idade: $(31.5 \pm 11.9)$ & 15 minutos de observação & & & & \\
\hline \multirow{5}{*}{ Routledge HC et al., $2002^{22}$} & 14 voluntários. 10 jovens & Os indivíduos foram avaliados através da VFC & & & & \\
\hline & 4 transplantados cardíacos. & 45 minutos de observação & $=\mathrm{PA}$ & $\mathrm{FC} \downarrow$ & $\mathrm{AF} \uparrow$ & NA \\
\hline & $\begin{array}{l}\text { Idade (24 a } 34 \text { anos) } \\
6 \text { homens } \\
\end{array}$ & & $p>0,05$ & $p<0,01$ & $p<0.01$ & \\
\hline & $\mathrm{GC}=20 \mathrm{ml}$ & & & & & \\
\hline & $\mathrm{Gl}=500 \mathrm{ml}$ & & & & & \\
\hline \multirow{5}{*}{ Chu YH et al., $2013^{31}$} & 22 voluntários & $\mathrm{GC}=50 \mathrm{ml}$ & & & & \\
\hline & & $\mathrm{Gl}=$ ingestão $500 \mathrm{ml}$ & $=\mathrm{PA}$ & FC $\downarrow$ & NA & $=\mathrm{RVP}$ \\
\hline & GC= 4 voluntários & $\begin{array}{l}\text { Foi coletada a amostragem sanguínea } 5 \text { minutos antes, } 25 \text { e } 50 \\
\text { minutos após a ingestão de água. }\end{array}$ & & & & \\
\hline & $\mathrm{Gl}=18$ voluntários & 50 minutos de observação & $p>0,05$ & $p<0,05$ & & $p>0,05$ \\
\hline & Idade (19 a 27 anos) & & & & & \\
\hline \multirow{4}{*}{ Schroeder C et al., $2002^{14}$} & 13 voluntários & $\begin{array}{l}\text { A quantidade de água pré-determinada foi ingerida } 15 \text { antes } \\
\text { do teste de inclinação de cabeça. }\end{array}$ & & & & \\
\hline & (Idade $31 \pm 3$ anos) & 25 minutos de observação & $\mathrm{PA} \uparrow$ & $F C \downarrow$ & $\mathrm{AF} \uparrow$ & $\operatorname{RVP} \uparrow$ \\
\hline & $\mathrm{GC}=50 \mathrm{ml}$ & & $p<0,05$ & $p<0,001$ & $p<0,05$ & $p<0,05$ \\
\hline & $\mathrm{Gl}=500 \mathrm{ml}$ & & & & & \\
\hline \multirow{4}{*}{ Lu CC et al., $2003^{16}$} & 22 voluntários & $\begin{array}{l}\text { Os indivíduos foram submetidos ao teste de inclinação a } 60^{\circ}, \mathrm{Gl}, \\
\text { ingerindo água } 5 \text { minutos antes do teste. }\end{array}$ & & & & \\
\hline & Idade (18 a 42 anos) & -45 minutos de observação & NA & $\mathrm{FC} \downarrow$ & NA & RVP $\uparrow$ \\
\hline & GC=Sem água & & & $p<0,05$ & & $p<0,001$ \\
\hline & $\mathrm{Gl}=473 \mathrm{ml}$ & & & & & \\
\hline \multirow{6}{*}{ Claydon VE et al.,(2006) } & 9 voluntários & $\begin{array}{c}\text { Os indivíduos foram submetidos ao teste de inclinação e ingeriram } \\
\text { água } 15 \text { antes da inclinação, em dois dias separados. }\end{array}$ & & & & \\
\hline & & 30 minutos de observação & $\mathrm{PA} \uparrow$ & $F C \downarrow$ & NA & $\operatorname{RVP} \uparrow$ \\
\hline & idade (36.8 \pm 4.2 anos) & & & & & \\
\hline & & & $\mathrm{p}<0,05$ & $p<0,05$ & & $\mathrm{p}<0,05$ \\
\hline & $\mathrm{GC}=50 \mathrm{ml}$ & & & & & \\
\hline & $\mathrm{Gl}=500 \mathrm{ml}$ & & & & & \\
\hline \multirow{5}{*}{ Jormeus A, et al., (2010) $)^{34}$} & 20 voluntários & & & & & \\
\hline & 10 homens & Duas semanas de observação. & $\mathrm{PA} \uparrow$ & NA & $\mathrm{NA}$ & NA \\
\hline & Idade $(23 \pm 2.0)$ & & & & & \\
\hline & GC= ingestão regular de água & & $p<0.005$ & & & \\
\hline & $\begin{array}{c}\mathrm{Gl}=\text { encorajados aumentar a ingestão em } \\
2 \text { litros de áqua. }\end{array}$ & & & & & \\
\hline \multirow{5}{*}{ Boschmann M et al., (2007) ${ }^{38}$} & 16 voluntários & 30 minutos em repouso. & & & & \\
\hline & 8 homens & 90 minutos de observação após ingerir as substâncias. & $=\mathrm{PA}$ & $=\mathrm{FC}$ & NA & NA \\
\hline & Idade: (20 a 42 anos) & & & & & \\
\hline & $\mathrm{GC}=50 \mathrm{ml}$ de água & & $p>0,05$ & $p>0,05$ & & \\
\hline & $\begin{array}{c}\mathrm{Gl}=500 \mathrm{ml} \text { de água; } 500 \mathrm{ml} \text { de solução } \\
\text { salina }\end{array}$ & & & & & \\
\hline \multirow{6}{*}{ Rylander R et al., (2004) ${ }^{33}$} & 70 indivíduos & Divididos em três grupos: & & & & \\
\hline & Homens e mulheres com idade entre & A) Água com baixa quantidade de minerais. & $\mathrm{PA} \uparrow$ & NA & NA & NA \\
\hline & (45-64 anos) & B) Água enriquecida com minerais & & & & \\
\hline & & C) Água mineral natural & $p<0,05$ & & & \\
\hline & & 1 litro por dia & & & & \\
\hline & & $\begin{array}{l}\text { Observação antes da ingestão, duas semanas depois e quatro } \\
\text { semanas após a ingestão. }\end{array}$ & & & & \\
\hline
\end{tabular}


A Figura 2 em florest-plot evidencia a análise do efeito da ingestão de água sobre a frequência cardíaca, evidenciando queda desta variável. Os resultados da esquerda indicam os valores favoráveis à influência da água na queda da FC quando comparada com o grupo controle, sendo o efeito combinado representado pelo losango. O teste de heterogeneidade aplicado na análise foi significativo ( $p=0,0001$ ), evidenciando estatisticamente a existência de heterogeneidade entre os estudos. Todos os estudos incluídos nesta meta-análise investigaram a possibilidade de efeitos da ingestão da água sobre a FC comparando valores de aproximadamente $500 \mathrm{~mL}$ para o grupo tratamento versus $50 \mathrm{~mL}$ de água para o grupo controle.

\section{DISCUSSÃO}

Nossos resultados ratificam a premissa que a IA influencia as atividades hemodinâmicas e autonômicas cardiovasculares, alterando a resistência vascular periférica, pressão arterial, frequência cardíaca e atividade vagal cardíaca ${ }^{14,16,22,25,30-34}$.

Os mecanismos envolvidos capazes de realizar alterações autonômicas e hemodinâmicas após a IA vêm sendo alvo de grandes estudos. No entanto, a distensão gástrica ${ }^{18,19}$ e a osmolalidade ${ }^{20}$ são duas vertentes importantes que buscam explicar estes fenômenos. A primeira evidencia que após a IA ocorra estimulação dos mecanorreceptores presentes no estômago, ocasionando aumento da atividade nervosa simpática muscular através de fibras aferentes do nervo esplâncnico ${ }^{35}$. Todavia, a teoria da osmolalidade vem sendo alvo de grandes estudos e mostram que a IA é capaz de estimular o receptor Trpv4, localizados no fígado e na circulação portal. Porém, toda a população dos receptores e os mecanismos de transdução ainda não estão bem esclarecidos, sendo aqueles sensíveis à queda de osmolalidade, no qual realizam um aumento reflexo da atividade simpática nervosa muscular, através do estímulo na atividade adrenérgica pós-ganglionar ${ }^{21}$.

Baseado nesses pressupostos há espaço para inferir que a IA deve ser controlada tanto na prática clínica quanto aos estudos que determinam verificar as variáveis autonômicas e hemodinâmicas.

Nossos estudos verificaram que os efeitos da IA apresentam resultados conflitantes no que diz respeito à alteração da pressão arterial, e a idade, as condições clínicas e o tipo de substância ingerida apresentam um forte fator para alterar esta variáve ${ }^{22,25,33}$.

O estudo de Routledgeet al. ${ }^{22}$ evidenciou que não houve alteração da pressão arterial em jovens saudáveis após a ingestão de $500 \mathrm{~mL}$ de água. Porém, foi observado aumento desta variável nos indivíduos transplantados cardíacos após a ingestão da mesma quantidade de água. Essa informação entra em consonância com os resultados vistos por outros autores ${ }^{25}$, os quais não constataram alteração da pressão arterial nos indivíduos jovens saudáveis do sexo masculino depois de ingerirem $500 \mathrm{~mL}$ de água. Todavia, outro fator foi levado em conta no estudo conduzido por outros autores ${ }^{33}$, observou que nos indivíduos com baixa quantidade de magnésio e cálcio, a água mineral e a água enriquecida de magnésio e outros minerais levaram uma diminuição significativa da pressão arterial nos indivíduos com hipertensão limítrofe, com faixa etária de 45 a 64 anos, visto após quatro semanas de observação.

Nossos resultados também evidenciam que a IA pode ser uma grande aliada no combate à hipotensão ortostática, tanto para indivíduos saudáveis quanto para aqueles que sofrem da doença ${ }^{14,30}$, podendo causar incremento da pressão arterial na posição de pé, após a ingestão de 500 mL de água, sendo este aumento da tolerância ortostática, evidenciado pelo teste de inclinação.

Em um estudo conduzido por Jordan et al. ${ }^{36}$ foi observado aumento da pressão arterial em indivíduos idosos e com insuficiência autonômica. Porém, não foi observado aumento da pressão arterial em indivíduos jovens.

Cabe aqui frisar que o aumento da atividade simpática nervosa sobre o tecido muscular parece explicar tal fenômeno, mas em indivíduos jovens, esse aumento está acompanhado por um incremento da atividade vagal cardíaca que, por sua vez, acredita-se agir contrapondo o aumento da pressão arterial22. Desta forma, este estímulo parece ser diminuído para indivíduos mais idosos e com insuficiência autonômica ${ }^{36}$.

A grande maioria dos estudos envolvidos nesta revisão, que investigaram os efeitos da IA sobre a frequência cardíaca, observaram diminuição desta variável ${ }^{14,22,25,31}$, estatisticamente significativo, evidenciado pela meta-análise $(p<0,001)$. Constatamos também em nossos resultados que a temperatura da água no momento da ingestão pode causar influências diretas na frequência cardíaca, evidenciando que a água gelada gera uma bradicardia pelo aumento da modulação vagal, isto quando comparado com o grupo que ingeriu água na temperatura ambiente ${ }^{32}$.

A bradicardia parece estar associada com mecanismos compensatórios para manutenção do débito cardíaco $^{20}$, sendo este mediado pela ativação da resposta parassimpática, após contrapor o aumento da atividade simpática nervosa sobre o tecido muscular. Já foi demonstrado que a estimulação vagal em humanos e em animais causa efeitos inotrópicos negativos, capazes de gerar diminuição da frequência cardíaca ${ }^{37}$.

Nesse mesmo contexto, os estudos que foram citados na presente revisão investigaram a atividade vagal cardíaca pela variabilidade da frequência cardíaca, e observaram aumento desta variável após a IA em indivíduos jovens em ambos os sexos ${ }^{14,22}$. Também vimos em nossos resultados que além da frequência cardíaca, a atividade parassimpática é mais ativada após a IA gelada ${ }^{32}$, sendo justificado por causar maior estímulo aos receptores Trpv4. Todavia, cabe ressaltar que outro estudo

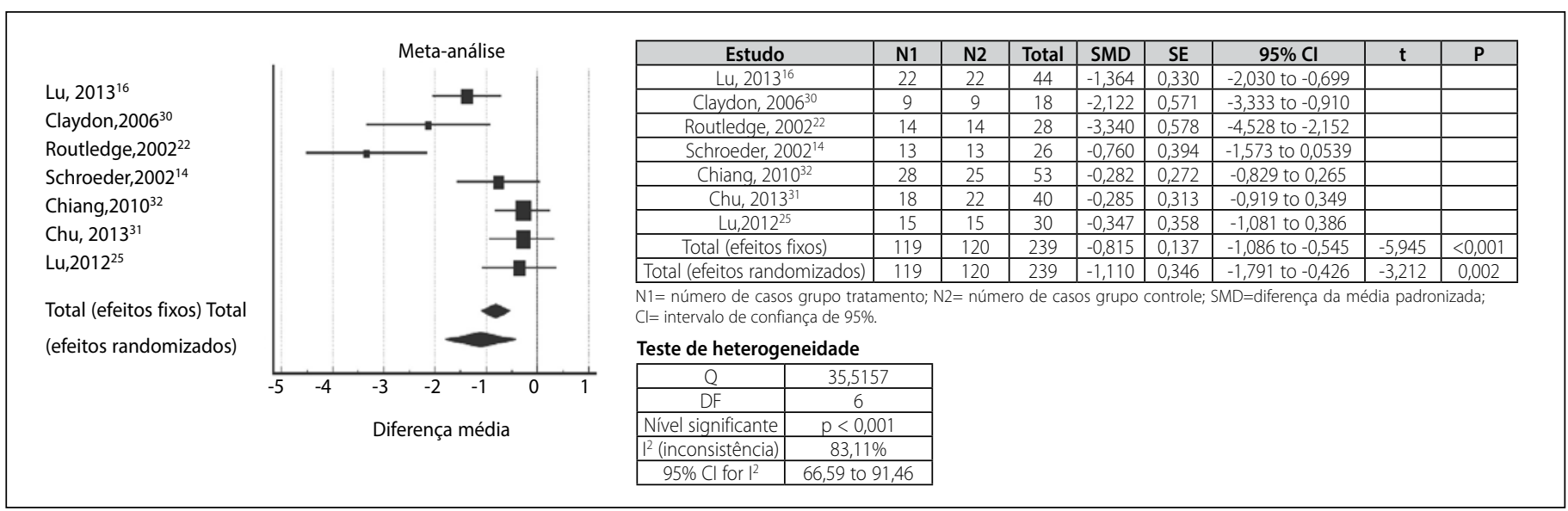

Figura 2. Forest Plot dos estudos incluídos na análise do efeito fixo e aleatório, diferença da média padronizada adotando intervalo de confiança de 95\%. 
que avaliou a IA em temperaturas diferentes nos indivíduos com insuficiência autonômica mostrou não haver alterações na pressão arterial após uma hora de ingestão ${ }^{36}$.

Os estudos que objetivaram investigar a resistência vascular periférica observaram aumento desta variável após a IA $14,16,25,30$.

Evidências suportam que este aumento seria importante para aliviar sintomas de indivíduos com síncope postural ${ }^{14,30}$, justificado por ocasionar aumento concomitante da pressão arterial. Lu et al. ${ }^{16}$, observaram incremento da tolerância ortostática em indivíduos jovens saudáveis e não somente nos indivíduos idosos e naqueles com alterações autonômicas, cuja resposta principal envolvida para este sucesso seria o aumento da resistência vascular periférica. Desta forma, Claydonetal ${ }^{30}$,observaram os efeitos da ingestão de $500 \mathrm{~mL}$ de água em pacientes com síncope postural, em que se verificou aumento na tolerância ortostática nesses voluntários, constatado pelo teste de inclinação. Os mecanismos responsáveis por gerar o aumento da resistência vascular periférica ainda não são completamente elucidados. Contudo, verifica-se que o aumento da atividade simpática nervosa sobre o tecido muscular, gerada através do estímulo da atividade adrenérgica pós-ganglionar, que por sua vez é ocasionada pela osmolalidade ou pela distensão gástrica, gera concomitante aumento da norepinefrina plasmática. Isso se observa, com maior frequência, em indivíduos idosos e com falência autonômica, acreditando-se que a pequena quantidade excretada de norepinefrina nesses grupos possa assumir papel importante na explicação deste fenômeno ${ }^{36}$.

Os estudos apresentam algumas limitações cujas interpretações e comparações podem ser prejudicadas, tais como: 1) design com baixa qualidade, por difícil realização de pesquisas com IA utilizando designs duplo-cego; 2) utilização de métodos distintos na avaliação dos fatores investigados, como o tempo de exposição após a IA bastantes variados.

\section{CONCLUSÃO}

Esta revisão confirma os efeitos da IA sobre o SNA em especial na FC, AF e RVP. Não obstante, as alterações hemodinâmicas expressas pela PA permanece ainda um óbice em relação à comunidade científica. Em adendo, este estudo mostra que o simples ato de ingerir água pode promover benefícios no tratamento da síncope postural, além da importância de controlar esta variável na prática clínica.

Todos os autores declararam não haver qualquer potencial conflito de interesses referente a este artigo.

CONTRIBUIÇÕES DOS AUTORES: Cada autor contribuiu individual e significantemente para o desenvolvimento deste artigo. TCR (0000-0003-1658-9713) contribuíram para concepção e delineamento da pesquisa. PSR (0000-0003-4035-3890) e DRR (0000-0003-2456-1470) tiveram papeis fundamentais na análise dos dados e suas interpretações. Na redação do manuscrito e aprovação da versão final a ser publicada, todos os autores tiveram contribuições significantes para. *Número ORCID (Open Researcher and Contributor ID).

\section{REFERÊNCIAS}

1. Kappers CU. The structure of the autonomic nervous system compared with its functional activity. J Physiol. 1908;37(2):139-45

2. Sharpey-Schafer E. The nomenclature of the autonomic nervous system. J Physiol. 1931;71(4):362-3.

3. Buch AN, Coote JH, Townend JN. Mortality, cardiac vagal control and physical training--what's the link? Exp Physiol. 2002;87(4):423-35

4. Cole CR, Blackstone EH, Pashkow FJ, Snader CE, Lauer MS. Heart-rate recovery immediately after exercise as a predictor of mortality. New Engl J Med. 1999;341(18):1351-7.

5. Jouven X, Empana JP, Schwartz PJ, Desnos M, Courbon D, Ducimetiere P. Heart-rate profile during exercise as a predictor of sudden death. New Engl J Med. 2005;352(19):1951-8.

6. Thayer JF, Lane RD. The role of vagal function in the risk for cardiovascular disease and mortality. Biol Psychol. 2007;74(2):224-42.

7. Araujo CG, Nobrega AC, Castro CL. Heart rate responses to deep breathing and 4-seconds of exercise before and after pharmacological blockade with atropine and propranolol. Clin Auton Res. 1992;2(1):35-40.

8. Bernardi L, Piepoli MF. Autonomic nervous system adaptation during physical exercise. Ital Heart J Suppl. 2001;2(8):831-9.

9. Elisberg El, Miller G, Weinberg SL, Katz LN. The effect of the Valsalva maneuver on the circulation. II. The role of the autonomic nervous system in the production of the overshoot. Am Heart J. 1953;45(2):227-36.

10. Hibino G, Moritani T, Kawada T, Fushiki T. Caffeine enhances modulation of parasympathetic nerve activity in humans: quantification using power spectral analysis. J Nutr. 1997;127(7):1422-7.

11. Vianna LC, Oliveira RB, Silva BM, Ricardo DR, Araujo CG. Water intake accelerates post-exercise cardiac vagal reactivation in humans. Eur J Appl Physiol. 2008;102(3):283-8.

12. Scott EM, Greenwood JP, Gilbey SG, Stoker JB, Mary DA. Water ingestion increases sympathetic vasoconstrictor discharge in normal human subjects. Clin Sci. 2001;100(3):335-42.

13. Callegaro CC, Moraes RS, Negrao CE, Trombetta IC, Rondon MU, Teixeira MS, et al. Acute water ingestion increases arterial blood pressure in hypertensive and normotensive subjects. J Hum Hypertens. 2007;21(7):564-70

14. Schroeder C, Bush VE, Norcliffe LJ, Luft FC, Tank J, Jordan J, et al. Water drinking acutely improves orthostatic tolerance in healthy subjects. Circulation. 2002;106(22):2806-11.

15. Mathias CJ, Young TM. Water drinking in the management of orthostatic intolerance due to orthostatic hypotension, vasovagal syncope and the postural tachycardia syndrome. Eur J Neurol. 2004;11(9):613-9.

16. Lu CC, Diedrich A, Tung CS, Paranjape SY, Harris PA, Byrne DW, et al. Water ingestion as prophylaxis against syncope. Circulation. 2003;108(21):2660-5.

17. Kapoor WN, Smith MA, Miller NL. Upright tilt testing in evaluating syncope: a comprehensive literature review. Am J Med. 1994;97(1):78-88.

18. van Orshoven NP, Oey PL, van Schelven $\sqcup$, Roelofs JM, Jansen PA, Akkermans LM. Effect of gastric distension on cardiovascular parameters: gastrovascular reflex is attenuated in the elderly. J Physiol. 2004;555(Pt 2):573-83.

19. Rossi P, Andriesse GI, Oey PL, Wieneke GH, Roelofs JM, Akkermans LM. Stomach distension increases efferent muscle sympathetic nerve activity and blood pressure in healthy humans. J Neurol Sci. 1998;161 (2):148-55.
20. Brown CM, Barberini L, Dulloo AG, Montani JP. Cardiovascular responses to water drinking: does osmolality play a role? Am J Physiol Regul Integr Comp Physiol. 2005;289(6):R1687-92.

21. May M, Jordan J. The osmopressor response to water drinking. Am J Physiol Regul Integr Comp Physiol. 2011;300(1):R40-6.

22. Routledge HC, Chowdhary S, Coote JH, Townend JN. Cardiac vagal response to water ingestion in normal human subjects. Clin Sci. 2002;103(2):157-62

23. Jordan J, Shannon JR, Grogan E, Biaggioni I, Robertson D. A potent pressor response elicited by drinking water. Lancet. 1999;353(9154):723.

24. Mendonca GV, Teixeira MS, Pereira FD, Fernhall B. Cardiovascular and autonomic effects of wate ingestion during postexercise circulatory occlusion. Appl Physiol Nutr Metab. 2012;37(6):1153-63.

25. Lu CC, Li MH, Lin TC, Chen TL, Chen RM, Tung CS, et al. Water ingestion reduces skin blood flow through sympathetic vasoconstriction. Clin Auton Res. 2012;22(2):63-9.

26. Shirreffs SM. Global patterns of water intake: how intake data affect recommendations. Nutr Rev. 2012;70(Suppl 2):S98-100.

27. Ma G, Zhang Q, Liu A, Zuo J, Zhang W, Zou S, et al. Fluid intake of adults in four Chinese cities. Nutr Rev. 2012;70(Suppl 2):S105-10.

28. Papadopoulos MC, Verkman AS. Aquaporin water channels in the nervous system. Nat Rev Neurosci. 2013;14(4):265-77.

29. Yool AJ. Aquaporins: multiple roles in the central nervous system. The Neuroscientist. 2007;13(5):470-85

30. Claydon VE, Schroeder C, Norcliffe LJ, Jordan J, Hainsworth R. Water drinking improves orthostatic tolerance in patients with posturally related syncope. Clin Sci. 2006;110(3):343-52.

31. Chu YH, Hsu YJ, Lee HS, Ho ST, Tung CS, Tseng CJ, et al. The osmopressor response is linked to upregulation of aquaporin-1 tyrosine phosphorylation on red blood cell membranes. Hypertension. 2013;62(1):197-202

32. Chiang CT, Chiu TW, Jong YS, Chen GY, Kuo CD. The effect of ice water ingestion on autonomic modulation in healthy subjects. Clin Auton Res. 2010;20(6):375-80.

33. Rylander R, Arnaud MJ. Mineral water intake reduces blood pressure among subjects with low urinary magnesium and calcium levels. BMC Public Health. 2004;4:56.

34. Jormeus A, Karlsson S, Dahlgren C, Lindstrom T, Nystrom FH. Doubling of water intake increase daytime blood pressure and reduces vertigo in healthy subjects. Clin Exp Hypertens. 2010;32(7):439-43.

35. Nosaka S, Murase S, Murata K. Arterial baroreflex inhibition by gastric distension in rats: mediation by splanchnic afferents. Am J Physiol. 1991;260(5 Pt 2):R985-94.

36. Jordan J, Shannon JR, Black BK, Ali Y, Farley M, Costa F, et al. The pressor response to water drinking in humans : a sympathetic reflex? Circulation. 2000;101(5):504-9.

37. Lewis ME, Al-Khalidi AH, Bonser RS, Clutton-BrockT, Morton D, Paterson D, et al. Vagus nerve stimulation decreases left ventricular contractility in vivo in the human and pig heart. J Physiol. 2001;534(Pt. 2):547-52

38. Boschmann M, Steiniger J, Franke G, Birkenfeld AL, Luft FC, Jordan J. Water drinking induces thermogenesis through osmosensitive mechanisms. J Clin Endocrinol Metab. 2007;92(8):3334-7. 